\title{
Convergence of a Block-By-Block Method for Nonlinear Volterra Integro-Differential Equations
}

\section{By Athena Makroglou*}

\begin{abstract}
The theory of a block-by-block method for solving Volterra integral equations is extended to nonsingular Volterra integro-differential equations. Convergence is proved and a rate of convergence is found. The convergence results obtained are analogous to those obtained by Weiss [12] for Volterra integral equations. Several numerical examples are included.
\end{abstract}

1. Introduction. Consider the nonlinear Volterra integro-differential equation

$$
y^{\prime}(x)=G\left(x, y(x), \quad \int_{0}^{x} K(x, t, y(t)) d t\right) \quad(x \geqslant 0)
$$

given $y(0)$. Methods applied to the integro-differential equation (1.1), seen as a differential equation, have been discussed by Linz [7], Brunner and Lambert [3], Tavernini [11], and Neves [10]. It is relevant to observe that the initial value problem (1.1) can be written in the form

$$
y(x)=\int_{0}^{x} G(s, y(s), z(s)) d s+y(0) \quad(x \geqslant 0)
$$

with

$$
z(x)=\int_{0}^{x} K(x, t, y(t)) d t \quad(x \geqslant 0)
$$

in which the initial condition is incorporated. If we put $\mathrm{F}(x, t, \varphi)=\left(F_{1}(t), F_{2}(t)\right)^{T}=$ $\left(G\left(t, \varphi_{1}, \varphi_{2}\right), K\left(x, t, \varphi_{1}\right)\right)^{T}$ with $\varphi=\left(\varphi_{1}, \varphi_{2}\right)^{T}$, then (1.2), (1.3) can be written

$$
\mathrm{f}(x)=\int_{0}^{x} \mathrm{~F}(x, s, \mathrm{f}(s)) d s+\mathrm{c} \quad(x \geqslant 0)
$$

where $\mathrm{f}(x)=(y(x), z(x))^{T}$ and $\mathrm{c}=(y(0), 0)^{T}$. Methods applied to the integro-differential equation (1.1), seen as an integral (or a coupled pair of integral) equation(s), have been discussed by Day [5], and Mocarsky [9].

In this paper we too shall consider the equation (1.1) written in the form (1.2), (1.3) and we shall extend a method first applied by Weiss [12] to Volterra integral

Received March 23, 1979; revised August 30, 1979.

AMS (MOS) subject classifications (1970). Primary 65R05; Secondary 65D05, 65D30.

* Formerly of the Department of Mathematics, University of Manchester, England. 
equations of the form

$$
f(x)=\int_{0}^{x} F(x, s, f(s)) d s+g(x) \quad(x>0) .
$$

This method is an implicit block-by-block method. Such methods have the advantage over linear multistep methods and step-by-step methods that they can be of high order and still be self starting. In a block-by-block method we seek approximate values of the solution for $0 \leqslant x_{m, j} \leqslant X$, where $x_{m, j}=m h+u_{j} h, j=0,1, \ldots, p, 0 \leqslant u_{0}<$ $u_{1}<\cdots<u_{p}, p$ integer, $m=0,1, \ldots, N-1$, such that $N h=X$.

Weiss [12] has derived two schemes based on interpolatory quadrature rules such that

$$
\int_{0}^{u_{j}} \varphi(x) d x \simeq \sum_{k=0}^{p} w_{k}^{j} \varphi\left(u_{k}\right)
$$

and

$$
\int_{0}^{1} \varphi(x) d x \simeq \sum_{k=0}^{p} w_{k} \varphi\left(u_{k}\right)
$$

where

$$
\begin{gathered}
w_{k}^{j}=\int_{0}^{u_{j}} L_{k}(x) d x, \\
w_{k}=w_{k}^{p}=\int_{0}^{1} L_{k}(x) d x,
\end{gathered}
$$

and

$$
L_{k}(x)=\prod_{j=0 ; j \neq k}^{p}\left(x-u_{j}\right) /\left(u_{k}-u_{j}\right)
$$

see Eqs. (2.11) and (2.12) in [12], that is

$$
\begin{aligned}
f_{m, j}= & h \sum_{i=0}^{m-1} \sum_{k=0}^{p} w_{k} F\left(x_{m, j}, x_{i, k}, f_{i, k}\right) \\
& +h \sum_{k=0}^{p} w_{k}^{j} F\left(x_{m, j}, x_{m, k}, f_{m, k}\right),
\end{aligned}
$$

and

$$
\begin{aligned}
f_{m, j}= & h \sum_{i=0}^{m-1} \sum_{k=0}^{p} w_{k} F\left(x_{m, j}, x_{i, k}, f_{i, k}\right) \\
& +h u_{j} \sum_{k=0}^{p} w_{k} F\left(x_{m, j}, x_{m, j, k}, \sum_{r=0}^{p} L_{r}\left(u_{j} u_{k}\right) f_{m, r}\right) .
\end{aligned}
$$

Scheme (1.11) has the disadvantage over scheme (1.12) that it needs the evaluation of $F(x, s, y(s))$ at points where $s>x$, where $F(x, s, y(s))$ might, for example, not be defined; see [12]. Weiss [12] indicated that the generalization of schemes (1.11), (1.12) 
to a system of Volterra integral equations of the second kind follows immediately. Equation (1.1) can be formulated as a system of Volterra integral equations of the form (1.4). But, because of the special form of $F_{1}(t)=G(t, y(t), z(t))$, simplifications occur and thus we chose to treat each of the equations (1.2), (1.3) separately. We so produced three schemes $\mathrm{A}, \mathrm{B}$, and $\mathrm{C}$, which we called block-by-block methods after Weiss, although they are new methods for integro-differential equations. We present here scheme $\mathrm{C}$ which in Makroglou [8] was proved to be the best of all three, namely the simplest, the least computer-time consuming and on the whole equally as accurate as $\mathrm{A}$ and $\mathrm{B}$. It also can be successfully used for Volterra integro-differential equations with weakly-singular kernels, as will be shown in a sequel to this; see also [8], scheme GC. The description of the scheme is given in Section 2 below. In Section 3 is given the convergence proof of scheme C. Some numerical results are included in Section 4. The results obtained by using schemes A, B, C in [8] were compared with those obtained by using linear multistep methods extended for the solution of (1.1) by Linz [7] and by Brunner and Lambert [3], hybrid methods applied to (1.1) by Makroglou [8] and some step-by-step methods discussed by Mocarsky [9]. We have not, however, undertaken a systematic assessment of the relative merits of the methods on a class of test problems. The results of the comparisons are stated in Section 4. For detailed results see [8].

Throughout the paper, $y_{m, j}$ shall denote an approximation to $y\left(x_{m, j}\right), m=$ $0,1, \ldots, N-1 ; j=0,1, \ldots, p$, and $z_{m, j}$ an approximation to $z\left(x_{m, j}\right)$. Also $x_{m, v_{0}, \ldots, v_{k}}$ shall denote the point $m h+u_{v_{0}} \cdots u_{v_{k}} h, 0 \leqslant v_{i} \leqslant p, i=0,1, \ldots, k$, $v_{i}$ integer.

\section{Description of the Method.}

2.1. Scheme C. Consider Eqs. (1.2), (1.3). For $x=x_{m, j}$ we have

$$
y\left(x_{m, j}\right)=\int_{0}^{x_{m}} G(s, y(s), z(s)) d s
$$

$$
+\int_{x_{m}}^{x_{m, j}} G(s, y(s), z(s))+y(0)
$$

and

$$
z\left(x_{m, j}\right)=\int_{0}^{x_{m}} K\left(x_{m, j}, t, y(t)\right) d t
$$

$$
\begin{aligned}
& +\int_{x_{m}}^{x_{m, j}} K\left(x_{m, j}, t, y(t)\right) d t \\
& \quad \quad m=0,1, \ldots, N-1 ; j=0,1, \ldots, p .
\end{aligned}
$$

Then using the quadrature rules (1.6), (1.8), (1.10) and (1.7), (1.9), (1.10) in the equations (2.1) and (2.2), respectively, we have 


$$
\begin{gathered}
y_{m, j}=y(0)+h \sum_{i=0}^{m-1} \sum_{k=0}^{p} w_{k} G\left(x_{i, k}, y_{i, k}, z_{i, k}\right) \\
+h \sum_{k=0}^{p} w_{k}^{j} G\left(x_{m, k}, y_{m, k}, z_{m, k}\right), \\
z_{m, j}=h \sum_{i=0}^{m-1} \sum_{k=0}^{p} w_{k} K\left(x_{m, j}, x_{i, k}, y_{i, k}\right) \\
+h u_{j} \sum_{k=0}^{p} w_{k} K\left(x_{m, j}, x_{m, j, k}, \sum_{r=0}^{p} L_{r}\left(u_{j} u_{k}\right) y_{m, r}\right), \\
m=0,1, \ldots, N-1 ; j=0,1, \ldots, p,
\end{gathered}
$$

where, to obtain an approximation of $y\left(x_{m, j, k}\right)$, we have used Lagrangian interpolation, that is,

$$
y\left(x_{m, j, k}\right) \simeq \sum_{r=0}^{p} L_{r}\left(u_{j} u_{k}\right) y\left(x_{m, r}\right)
$$

We note that in (1.2) $G(s, y(s), z(s))$ does not depend on $x$ and so the evaluation of $G$ at points where $s>x$ will not matter.

In the case when $u_{0}=0$ Eqs. (2.3) simplify to

$$
y_{m, j}=y_{m, 0}+h \sum_{k=0}^{p} w_{k}^{j} G\left(x_{m, k}, y_{m, k}, z_{m, k}\right) .
$$

(We cannot make a similar simplification to Eq. (2.4), because there the sum from 0 to $m-1$ is dependent on $j$.)

When $u_{0} \neq 0$ we can still simplify (2.3), by differencing on $m$ thus

$$
\begin{gathered}
y_{0, j}=s(0, j)+y(0), \\
y_{m, j}-h s(m, j)=y_{m-1, j}+h s(m-1, j) \\
+h \sum_{k=0}^{p} w_{k} G\left(x_{m-1, k}, y_{m-1, k}, z_{m-1, k}\right), \\
\quad m \geqslant 1 ; j=0,1, \ldots, p,
\end{gathered}
$$

where we have put

$$
s(m, j)=\sum_{k=0}^{p} w_{k}^{j} G\left(x_{m, k}, y_{m, k}, z_{m, k}\right), \quad m=0,1, \ldots, N-1 .
$$

At the $m$-stage in Eq. (2.7) $y_{m-1, j}, z_{m-1, j}$, and also $s(m-1, j)$ are known from the previous stage for $j=0,1, \ldots, p$, and we have to compute only one term of the sum from 0 to $m-1$ on the right-hand side of (2.3), namely the one for $i=m-1$. 
We may note that the scheme (Eqs. (2.3), (2.4)) is self starting and that for $p=1, u_{0}=0, u_{1}=1$, it reduces to the scheme obtained, if we apply the trapezium quadrature rule to the integrals occurring.

3. Convergence. In this section we are concerned with a convergence proof for scheme C (Eqs. (2.3), (2.4)).

We shall establish a bound on the error in approximations (2.3), (2.4), see Theorem 1. This bound is achieved in terms of certain discretization errors $\left(M_{1}(h)\right.$, $T_{1}(h), T_{2}(h), T_{3}(h), T_{4}(h)$ in the analysis to follow) by using a lemma [6, p. 313], given as Lemma 2 below. We shall then deduce convergence, see Corollary 1 , and the rate of convergence of the method by using Lemma 1 ([12], [1]), see Corollary 2 .

Before proceeding to the proofs, we need the following preliminaries; see Weiss [12]. In relation to the quadrature rules (1.6)-(1.10), we define

$$
g(x)=\left(x-u_{0}\right)\left(x-u_{1}\right) \cdots\left(x-u_{p}\right)=\sum_{j=0}^{p+1} c_{j} x^{p+1-j}, \quad c_{0}=1 .
$$

We denote the relation $\int_{0}^{1} g(x) d x \neq 0$ by $g(x) \in P_{0}$ and the relations $\int_{0}^{1} x^{i} g(x) d x=0$, $i=0,1, \ldots, v-1, \int_{0}^{1} x^{v} g(x) d x \neq 0$, by $g(x) \in P_{v}$. Let

$$
E_{j}(\varphi)=\int_{0}^{u_{j}} \varphi(x) d x-\sum_{k=0}^{p} w_{k}^{j} \varphi\left(u_{k}\right), \quad j=0,1, \ldots, p
$$

Then the following result is valid.

Lemma 1 ([12], [1], [8]). If $g(x) \in P_{v}$, then

$$
E_{j}\left(s^{p+1+\eta}\right)=\int_{0}^{u_{j}} x^{r} g(x) d x-\sum_{i=1}^{r} c_{i} E_{j}\left(s^{p+1+r-i}\right), \quad r=0,1, \ldots, p
$$

and

$$
E_{p}\left(s^{p+1+\eta}\right)=0 \text { for } r \leqslant v-1
$$

From (3.4) we may conclude that the degree of precision of the formula (1.7) is $p+1+v-1=p+v$. The following lemma gives an estimation of the growth of the solution of nonhomogeneous difference equations.

LEMMA 2 ([6, p. 313]). If $\left|q_{n}\right| \leqslant A \sum_{i=0}^{n-1}\left|q_{i}\right|+B$ for $n=s, s+1, \ldots$ with $A>0, B>0$ and $\Sigma_{i=0}^{s-1}\left|q_{i}\right| \leqslant P$, then $\left|q_{n}\right| \leqslant(B+A P)(1+A)^{n-s}, n=s, s+1, \ldots$ Furthermore, if $A=h k$ and $n h=x$, then $\left|q_{n}\right| \leqslant(B+h k P) \exp (k x)$.

We shall also make use of the following notation:

$$
\begin{gathered}
\Gamma(x) \equiv G(x, y(x), z(x)), \quad K_{1}(x, s) \equiv K(x, s, y(s)), \quad 0 \leqslant s \leqslant x \leqslant X, \\
\left.\Gamma^{(m)}(s) \equiv \frac{\partial^{m}}{\partial \eta^{m}} \Gamma(\eta)\right|_{\eta=s},\left.\quad K_{1}^{(m)}(x, s) \equiv \frac{\partial^{m}}{\partial \eta^{m}} K_{1}(x, \eta)\right|_{\eta=s}
\end{gathered}
$$


We now define

$$
\begin{aligned}
& L=\sup _{0 \leqslant r, j, k \leqslant p}\left|L_{r}\left(u_{j} u_{k}\right)\right|, \quad \Lambda_{p}=\left\|\sum_{j=0}^{p} L_{j}(x)\right\|_{\infty}, \\
& W=\sup _{0 \leqslant k<p}\left|w_{k}\right|, \quad W^{\prime}=\sup _{0 \leqslant j, k \leqslant p}\left|w_{k}^{j}\right|, \\
& \Delta_{1}=\{(x, y, z): 0 \leqslant x \leqslant X,|y|<\infty,|z|<\infty\}, \\
& \Delta_{2}=\{(x, s, y): 0 \leqslant s \leqslant x \leqslant X,|y|<\infty\}, \\
& M_{1}(h)=\max _{\substack{0<m<N-1 \\
0<j<p}}\left|\int_{0}^{x_{m}} K_{1}\left(x_{m, j}, s\right) d s-h \sum_{i=0}^{m-1} \sum_{k=0}^{p} w_{k} K_{1}\left(x_{m, j}, x_{i, k}\right)\right| \text {, } \\
& M_{2}(h)=\max _{0 \leqslant m \leqslant N-1}\left|\int_{0}^{x_{m}} \Gamma(x) d x-h \sum_{i=0}^{m-1} \sum_{k=0}^{p} w_{k} \Gamma\left(x_{i, k}\right)\right|, \\
& \begin{aligned}
T_{1}(h)= & \max _{\substack{0 \leqslant m \leqslant N-1 \\
0<j<p}} \mid \int_{x_{m}}^{x_{m, j}} K_{1}\left(x_{m, j}, s\right) d s \\
& \quad-h u_{j} \sum_{k=0}^{p} w_{k} K_{1}\left(x_{m, j}, x_{m, j, k}\right) \mid,
\end{aligned} \\
& T_{2}(h)=\max _{\substack{0 \leqslant m \leqslant N-1 \\
0<j<p}}\left|\int_{x_{m}}^{x_{m, j}} \Gamma(x) d x-h \sum_{k=0}^{p} w_{k}^{j} \Gamma\left(x_{m, k}\right)\right| \text {, } \\
& T_{3}(h)=\max _{\substack{0 \leqslant m \leqslant N-1 \\
0<j, k<p}}\left|y\left(x_{m, j, k}\right)-\sum_{r=0}^{p} L_{r}\left(u_{j} u_{k}\right) y\left(x_{m, r}\right)\right|, \\
& e_{m, j}=y\left(x_{m, j}\right)-y_{m, j}, \quad m=0,1, \ldots, N-1 ; j=0,1, \ldots, p, \\
& e_{m}=\max _{0 \leqslant j \leqslant p}\left|e_{m, j}\right|, \quad m=0,1, \ldots, N-1,
\end{aligned}
$$

and proceed to the proof of the convergence theorems.

THEOREM 1. Let $L_{1}, L_{2}$ be the Lipschitz constants of $G(x, y, z)$ with respect to the second and third variable, respectively, and $L_{3}$ the Lipschitz constant of $K(x, s, y)$ with respect to the third variable on the sets $\Delta_{1}, \Delta_{2}$. Then for the error $e_{m}$ in the approximations (2.3), (2.4) we have, for $h$ sufficiently small, that

$$
e_{m} \leqslant\left(C^{\prime}+h E^{\prime} \xi\right) \exp \left((m-1) h E^{\prime}\right), \quad m=1,2, \ldots, N-1,
$$

and

(3.9) $e_{0} \leqslant\left(T_{2}(h)+h W^{\prime} L_{2}(p+1)\left[T_{1}(h)+h W L_{3}(p+1) T_{3}(h)\right]\right) /(1-D(p+1))$, where

$$
C^{\prime}=C /(1-D(p+1)), \quad E^{\prime}=E(p+1) /(1-D(p+1))
$$




$$
\begin{aligned}
C= & M_{2}(h)+W L_{2} X(p+1)\left(M_{1}(h)+T_{1}(h)\right)+h W^{2} L_{2} L_{3} X(p+1)^{2} T_{3}(h) \\
& +T_{2}(h)+h W^{\prime} L_{2}(p+1)\left[M_{1}(h)+T_{1}(h)+h W L_{3}(p+1) T_{3}(h)\right], \\
D= & h W^{\prime} L_{1}+h^{2} W W^{\prime} L_{2} L_{3} L(p+1)^{2} \\
E= & W L_{1}+W^{2} L_{2} L_{3} X(p+1)+h W^{2} L_{2} L_{3} L(p+1)^{2}+h W W^{\prime} L_{2} L_{3}(p+1),
\end{aligned}
$$

and $\xi$ is such that $e_{0} \leqslant \xi$.

Proof. Subtracting (2.3) from (2.1) we find

$$
e_{m, j}=A+B
$$

where $A \equiv A_{m}, B \equiv B_{m, j}$ with

$$
A=\int_{0}^{x_{m}} \Gamma(x) d x-h \sum_{i=0}^{m-1} \sum_{k=0}^{p} w_{k} G\left(x_{i, k}, y_{i, k}, z_{i, k}\right),
$$

$$
B=\int_{x_{m}}^{x_{m, j}} \Gamma(x) d x-h \sum_{k=0}^{p} w_{k}^{j} G\left(x_{m, k}, y_{m, k}, z_{m, k}\right),
$$

and $z_{i, k}$ is the approximation to the integral $z\left(x_{i, k}\right)=\int_{0}^{x_{i, k}} K_{1}\left(x_{i, k}, s\right) d s, i=0$, $1, \ldots, m ; k=0,1, \ldots, p$, given from Eq. (2.4) by

$$
\begin{aligned}
z_{i, k}= & h \sum_{\lambda=0}^{i-1} \sum_{\mu=0}^{p} w_{\mu} K\left(x_{i, k}, x_{\lambda, \mu}, y_{\lambda, \mu}\right) \\
& +h u_{k} \sum_{\mu=0}^{p} w_{\mu} K\left(x_{i, k}, x_{i, k, \mu}, \sum_{r=0}^{p} L_{r}\left(u_{k} u_{\mu}\right) y_{i, r}\right)
\end{aligned}
$$

We shall proceed using the "add and subtract" procedure; see, for example, Mocarsky [9]. So if we denote by $z^{\prime}\left(x_{i, k}\right)$ the right-hand side of (3.14) with $y_{\lambda, \mu}, y_{i, r}$ replaced by $y\left(x_{\lambda, \mu}\right), y\left(x_{i, r}\right)$, we have

$$
\left|e_{m, j}\right| \leqslant \sum_{i=0}^{4}\left|A_{i}\right|+\sum_{i=0}^{4}\left|B_{i}\right|
$$

where

$$
\begin{aligned}
& A_{1}=\int_{0}^{x_{m}} \Gamma(x) d x-h \sum_{i=0}^{m-1} \sum_{k=0}^{p} w_{k} \Gamma\left(x_{i, k}\right), \\
& A_{2}=h \sum_{i=0}^{m-1} \sum_{k=0}^{p} w_{k}\left[\Gamma\left(x_{i, k}\right)-G\left(x_{i, k}, y\left(x_{i, k}\right), z^{\prime}\left(x_{i, k}\right)\right)\right], \\
& A_{3}=h \sum_{i=0}^{m-1} \sum_{k=0}^{p} w_{k}\left[G\left(x_{i, k}, y\left(x_{i, k}\right), z^{\prime}\left(x_{i, k}\right)\right)-G\left(x_{i, k}, y_{i, k}, z^{\prime}\left(x_{i, k}\right)\right)\right], \\
& A_{4}=h \sum_{i=0}^{m-1} \sum_{k=0}^{p} w_{k}\left[G\left(x_{i, k}, y_{i, k}, z^{\prime}\left(x_{i, k}\right)\right)-G\left(x_{i, k}, y_{i, k}, z_{i, k}\right)\right],
\end{aligned}
$$


and

$$
\begin{aligned}
& B_{1}=\int_{x_{m}}^{x_{m, j}} \Gamma(x) d x-h \sum_{k=0}^{p} w_{k}^{j} \Gamma\left(x_{m, k}\right), \\
& B_{2}=h \sum_{k=0}^{p} w_{k}^{j}\left[\Gamma\left(x_{m, k}\right)-G\left(x_{m, k}, y\left(x_{m, k}\right), z^{\prime}\left(x_{m, k}\right)\right)\right],
\end{aligned}
$$

$$
\begin{aligned}
& B_{3}=h \sum_{k=0}^{p} w_{k}^{j}\left[G\left(x_{m, k}, y\left(x_{m, k}\right), z^{\prime}\left(x_{m, k}\right)\right)-G\left(x_{m, k}, y_{m, k}, z^{\prime}\left(x_{m, k}\right)\right)\right], \\
& \left.B_{4}=h \sum_{k=0}^{p} w_{k}^{j}\left[G\left(x_{m, k}, y_{m, k}, z^{\prime}\left(x_{m, k}\right)\right)-G\left(x_{m, k}, y_{m, k}, z_{m, k}\right)\right)\right] .
\end{aligned}
$$

Using the Lipschitz conditions on $G$ and $K$, the definitions (3.7) and the triangle inequality, we obtain the following inequalities (see [8, pp. 178, 179, 197]),

$$
\begin{aligned}
& \left|A_{1}\right| \leqslant M_{2}(h), \\
& \left|A_{2}\right| \leqslant W L_{2} X(p+1)\left(M_{1}(h)+T_{1}(h)\right)+h W^{2} L_{2} L_{3} X(p+1)^{2} T_{3}(h), \\
& \left|A_{3}\right| \leqslant h W L_{1} \sum_{i=0}^{m-1} \sum_{k=0}^{p}\left|e_{i, k}\right|
\end{aligned}
$$

$$
\begin{aligned}
\left|A_{4}\right| \leqslant & h W^{2} L_{2} L_{3} X(p+1) \sum_{\lambda=0}^{m-1} \sum_{\mu=0}^{p}\left|e_{\lambda, \mu}\right| \\
& +h^{2} W^{2} L_{2} L_{3} L(p+1)^{2} \sum_{i=0}^{m-1} \sum_{r=0}^{p}\left|e_{i, r}\right|
\end{aligned}
$$

and

$$
\begin{aligned}
\left|B_{1}\right| & \leqslant T_{2}(h), \\
\left|B_{2}\right| & \leqslant h W^{\prime} L_{2}(p+1)\left[M_{1}(h)+T_{1}(h)+h W L_{3}(p+1) T_{3}(h)\right], \\
\left|B_{3}\right| & \leqslant h W^{\prime} L_{1} \sum_{k=0}^{p}\left|e_{m, k}\right|, \\
\left|B_{4}\right| & \leqslant h W^{\prime} L_{2}(p+1)\left[h W L_{3} \sum_{i=0}^{m-1} \sum_{r=0}^{p}\left|e_{i, r}\right|+h W L_{3} L(p+1) \sum_{r=0}^{p}\left|e_{m, r}\right|\right] .
\end{aligned}
$$

From (3.15), (3.18), (3.19) we then find

$$
\left|e_{m, j}\right| \leqslant C+D \sum_{r=0}^{p}\left|e_{m, r}\right|+h E \sum_{i=0}^{m-1} \sum_{r=0}^{p}\left|e_{i, r}\right|
$$

or

$$
e_{m} \leqslant C^{\prime}+h E^{\prime} \sum_{i=0}^{m-1} e_{i}
$$


Applying Lemma 2 now to (3.21) for $h$ sufficiently small and $e_{0} \leqslant \xi$ we obtain the result (3.8) of the theorem. The result (3.9) can be proved proceeding similarly and taking into account the fact that for $m=0$ we have $M_{1}(h)=0$ and $M_{2}(h)=0$.

Using the result of Theorem 1, we shall now establish convergence; that is, we shall prove

Corollary 1. Let

(i) the assumptions of Theorem 1 be valid,

(ii) $y(x), z(x), G(x, y(x), z(x)), K(x, s, y(s))$ be continuous for $x \in[0, X]$, $s \in[0, x]$, and

(iii) $g(x)=\left(x-u_{0}\right)\left(x-u_{1}\right) \cdots\left(x-u_{p}\right) \in P_{v}$.

Then $e_{m} \rightarrow 0, m \leqslant N$ as $h \longrightarrow 0, N h=X$.

Proof. We shall prove that

$$
\begin{aligned}
\lim _{h \rightarrow 0} M_{1}(h) & =\lim _{h \rightarrow 0} M_{2}(h)=\lim _{h \rightarrow 0} T_{1}(h)=\lim _{h \rightarrow 0} T_{2}(h) \\
& =\lim _{h \rightarrow 0} T_{3}(h)=0, \quad N h=X,
\end{aligned}
$$

and then from (3.8), (3.9) the required result follows.

From Lemma 1 we have that the degree of precision of the quadrature rule (1.7) is $p+v \geqslant p$. Thus,

$$
M_{2}(h) \leqslant 6 X\left(1+\Lambda_{p}\right) \omega(\Gamma ; h / 2 p) \quad([8, \text { result I-2.20]), }
$$

where $\Lambda_{p}$ is defined in (3.7) and

$$
\omega(\varphi ; \delta)=\sup _{\substack{x_{1}, x_{2} \in[a, b] \\\left|x_{1}-x_{2}\right| \leqslant \delta}}\left|\varphi\left(x_{1}\right)-\varphi\left(x_{2}\right)\right|
$$

is the modulus of continuity of a function $\varphi(x)$. So $\lim _{h \rightarrow 0 ; N h=X} M_{2}(h)=0$. Also,

$$
M_{1}(h) \leqslant 6 X\left(1+\Lambda_{p}\right) \max _{\substack{0 \leqslant m \leqslant N-1 \\ 0<j<p}} \omega\left(K_{1}\left(x_{m, j}, s\right) ; h / 2 p\right),
$$

where $K_{1}(x, s) \equiv K(x, s, y(s)), 0 \leqslant s \leqslant x \leqslant X$, is uniformly continuous in $0 \leqslant s \leqslant x$ $\leqslant X$. So

$$
\max _{\substack{0 \leqslant m \leqslant N-1 \\ 0<j<p}} \omega\left(K_{1}\left(x_{m, j}, s\right) ; h / 2 p\right) \rightarrow 0 \quad \text { as } h \rightarrow 0,
$$

which implies that $\lim _{h \rightarrow 0 ; N h=X} M_{1}(h)=0$. For $T_{1}(h)$ we have

$$
T_{1}(h) \leqslant 6 h\left(1+\Lambda_{p}\right) \max _{\substack{0 \leqslant m \leqslant N-1 \\ 0<j<p}} \omega\left(K_{1}\left(x_{m, j}, m h+u_{j} h s\right) ; 1 / 2 p\right),
$$

and therefore $\lim _{h \rightarrow 0 ; N h=X} T_{1}(h)=0$. For $T_{2}(h)$, since the degree of precision of rule (1.6) is $\geqslant 0$ and the continuity assumption of $\Gamma(x)$ on $0 \leqslant x \leqslant X$ implies the 
bounded and Riemann integrable of $\Gamma(x)$, we have $\lim _{h \rightarrow 0 ; N h=X} T_{2}(h)=0$; see [5, p. 25]. Finally, for the error $T_{3}(h)$ in Lagrangian interpolation, using result (i-2.7) and (i) of (Jackson) Theorem I-2 in [8] we have $T_{3}(h) \leqslant 6\left(1+\Lambda_{p}\right) \omega(y ; h / 2 p)$. $y(x) \in C([0, X])$ implies that $y(x)$ is uniformly continuous in $[0, X],(X<\infty)$. Thus, $\lim _{h \rightarrow 0 ; N h=X} \omega(y ; h / 2 p)=0$ and so $\lim _{h \rightarrow 0 ; N h=X} T_{3}(h)=0$.

Having examined convergence, we shall examine the rate of convergence; that is, we shall prove

\section{Corollary 2. Let}

(i) the assumptions of Theorem 1 be valid,

(ii) $g(x) \in P_{v}$,

(iii) $K(x, s, y)$ is $p+v+2$ times continuously differentiable with respect to $x$, $s$ and $y$, respectively, on $0 \leqslant s \leqslant x, 0 \leqslant x \leqslant X,|y| \leqslant \bar{y}$, where $\bar{y}=\max _{0<x<X}|y(x)|$,

(iv) $G(x, y, z)$ is $p+v+2$ times continuously differentiable with respect to $x$, $y$ and $z$, respectively on $0 \leqslant x \leqslant X,|y| \leqslant \bar{y},|z| \leqslant \bar{z}$, where $\bar{y}$ is as in (iii) and $\bar{z}=$ $\max _{0<x \leqslant X}|z(x)|$,

(v) $y(x)$ is $p+2$ times continuously differentiable on $0 \leqslant x \leqslant X$.

Then, there are constants $C_{1}, C_{2}, C_{3}$ such that,

$$
\begin{array}{rlrl}
e_{0} & \leqslant C_{1} h^{p+2}, & & \\
e_{m} & \leqslant C_{2} h^{p+1}, & m=1,2, \ldots, N-1, \text { if } v=0, \\
e_{m} & \leqslant C_{3} h^{p+2}, & m=1,2, \ldots, N-1, \text { if } v>0 .
\end{array}
$$

Proof. Consider the definitions in (3.5), (3.6). Then for the errors $M_{2}(h)$, $M_{1}(h), T_{1}(h), T_{2}(h)$ in (3.7), extending the analysis of Weiss [12], we find

$$
M_{2}(h)=\max _{0 \leqslant m \leqslant N-1} \frac{h^{p+v+2}}{(p+v+1) !}\left|E_{p}\left(s^{p+v+1}\right) \sum_{i=0}^{m-1} \Gamma^{(p+v+1)}\left(x_{i}\right)\right|+O\left(h^{p+v+3}\right)
$$

or

$$
\begin{aligned}
M_{2}(h)= & \max _{0 \leqslant m \leqslant N-1} \frac{h^{p+v+1}}{(p+v+1) !}\left|E_{p}\left(s^{p+v+1}\right) \int_{0}^{x_{m}} \Gamma^{(p+v+1)}(s) d s\right| \\
& +O\left(h^{p+v+2}\right)
\end{aligned}
$$

where we have used Taylor series expansion for $\Gamma\left(x_{i}+h s\right), \Gamma\left(x_{i}+u_{k} h\right)$ and that $E_{p}\left(s^{p+1+r}\right)=0$ if $r \leqslant v-1$ (Lemma 1). Similarly we find

$$
\begin{aligned}
M_{1}(h)= & \max _{\substack{0<m<N-1 \\
0<j<p}} \frac{h^{p+v+1}}{(p+v+1) !}\left|E_{p}\left(s^{p+v+1}\right) \int_{0}^{x_{m, j}} K_{1}^{(p+v+1)}\left(x_{m, j}, s\right) d s\right| \\
& +O\left(h^{p+v+2}\right) .
\end{aligned}
$$

For $T_{2}(h)$, using Taylor series expansion for $\Gamma\left(x_{m, j}+h\left(s-u_{j}\right)\right)$ and 
$\Gamma\left(x_{m, j}+h\left(u_{k}-u_{j}\right)\right)$, we find

$$
T_{2}(h)=\max _{0 \leqslant m \leqslant N-1} h\left|\sum_{r=p+1}^{p+v} \frac{h^{r}}{r !} \Gamma^{(r)}\left(x_{m, j}\right) E_{j}\left(\left(s-u_{j}\right)^{r}\right)\right|+O\left(h^{p+v+2}\right)
$$

$\left(E_{i}\left(s^{r}\right)=0\right.$ for $\left.r=0,1, \ldots, p\right)$.

For $T_{1}(h)$, using Taylor series expansion for $K_{1}\left(x_{m, j}, x_{m, j}+u_{j} h(s-1)\right)$ and $K_{1}\left(x_{m, j}, x_{m, j}+u_{j} h\left(u_{k}-1\right)\right)$, we find

$$
\begin{aligned}
T_{1}(h)= & \max _{\substack{0 \leqslant m \leqslant N-1 \\
0<j<p}} u_{j} h\left|\sum_{r=p+1}^{p+v} \frac{u_{j}^{r} h^{r}}{r !} K_{1}^{(r)}\left(x_{m, j}, x_{m, j}\right) E_{p}\left((s-1)^{\eta}\right)\right| \\
& +O\left(h^{p+v+2}\right),
\end{aligned}
$$

$\left(E_{i}\left(s^{r}\right)=0\right.$ for $\left.r=0,1, \ldots, p\right)$.

For the Lagrangian interpolation, using the well-known error formula, we have

$$
T_{3}(h)=\max _{\substack{0 \leqslant m<N-1 \\ 0 \leqslant j, k \leqslant p}} h^{p+1}\left|y^{(p+1)}(\zeta) g\left(u_{j} u_{k}\right)\right| /(p+1) !+O\left(h^{p+2}\right),
$$

where $\zeta$ lies between $x_{m, 0}, x_{m, p}$ and $x_{m, j, k}$.

Combining now the results above with (3.8), (3.9), we obtain the required result (3.23).

The same rate of convergence was found in [8] for the other two schemes mentioned in the introduction, but for scheme $\mathrm{A}$ under the additional assumption that $z(x)$ is $p+2$ times continuously differentiable on $0 \leqslant x \leqslant X$.

4. Numerical Results. We now display some numerical results obtained by testing scheme $C$ on three examples ((a), (b), (c) below) for the cases $p=2, p=3$, $u_{0}=0, u_{i}=i / p, i=1, \ldots, p$, with $v=1$ and $v=0$, respectively. The results verify order of convergence $O\left(h^{p+2}\right)=O\left(h^{4}\right)$ and $O\left(h^{p+1}\right)=O\left(h^{4}\right)$, respectively. Example (c) is a "stiff" (constructed) example. In general, Eqs. (2.3), (2.4) form a nonlinear system for $y_{m, 0}, y_{m, 1}, \ldots, y_{m, p} ; z_{m, 0}, z_{m, 1}, \ldots, z_{m, p}$, which we solved by applying a Newton iteration. It might seem preferable to use the method with $z_{m, 0}, \ldots, z_{m, p}$ eliminated (see [8], methods "with elimination of the $z$-variable") in order to reduce computing time; a sample of actual computing time (see [8, p. 146]) though, shows this to be false.

(a) $y(x)=1+y(x)-x \exp \left(-x^{2}\right)-2 \int_{0}^{x} x s \exp \left(-y^{2}(s)\right) d s, 0 \leqslant x \leqslant 1, y(0)=0$, $y(x)=x$ (Mocarsky [9, p. 239], Linz [7, p. 301], Makroglou [8, Example 2, p. 93]).

(b) $y^{\prime}(x)=1+2 x-y(x)+\int_{0}^{x} x(1+2 x) \exp (s(x-s)) y(s) d s, 0 \leqslant x \leqslant 1, y(0)=$ 1, $y(x)=\exp \left(x^{2}\right)(\operatorname{Linz}[7$, p. 300], Makroglou [8, Example 6, p. 94]).

(c) $y^{\prime}(x)=y^{\prime}(0)+\int_{0}^{x}-20 y(s) d s+21 y(0)-21 y(s), y(0)=1, y^{\prime}(0)=1$, $y(x)=(21 / 19) \exp (-x)-(2 / 19) \exp (-20 x)$ (Makroglou [8, Example 7b, pp. 94, 95]). 
Example (a) Scheme C

\begin{tabular}{lllll}
\multicolumn{4}{c}{$u_{0}=0, u_{i}=i / p$} & $(i=1,2, \ldots, p)$ \\
\hline & \multicolumn{2}{c}{$p=2$} & \multicolumn{2}{c}{$p=3$} \\
\cline { 2 - 5 } & $h=0.1$ & $h=0.05$ & $h=0.1$ & $h=0.05$ \\
\hline 0.1 & $3.56 \times 10^{-11}$ & $2.26 \times 10^{-12}$ & $1.38 \times 10^{-11}$ & $9.63 \times 10^{-13}$ \\
0.2 & $5.77 \times 10^{-10}$ & $4.29 \times 10^{-11}$ & $2.46 \times 10^{-10}$ & $1.88 \times 10^{-11}$ \\
0.3 & $3.22 \times 10^{-9}$ & $2.29 \times 10^{-10}$ & $1.41 \times 10^{-9}$ & $1.01 \times 10^{-10}$ \\
0.4 & $1.06 \times 10^{-8}$ & $7.29 \times 10^{-10}$ & $4.66 \times 10^{-9}$ & $3.22 \times 10^{-10}$ \\
0.5 & $2.59 \times 10^{-8}$ & $1.74 \times 10^{-9}$ & $1.14 \times 10^{-8}$ & $7.73 \times 10^{-10}$ \\
0.6 & $5.26 \times 10^{-8}$ & $3.48 \times 10^{-9}$ & $2.32 \times 10^{-8}$ & $1.54 \times 10^{-9}$ \\
0.7 & $9.37 \times 10^{-8}$ & $6.13 \times 10^{-9}$ & $4.15 \times 10^{-8}$ & $2.72 \times 10^{-9}$ \\
0.8 & $1.52 \times 10^{-7}$ & $9.85 \times 10^{-9}$ & $6.73 \times 10^{-8}$ & $4.37 \times 10^{-9}$ \\
0.9 & $2.29 \times 10^{-7}$ & $1.48 \times 10^{-8}$ & $1.02 \times 10^{-7}$ & $6.56 \times 10^{-9}$ \\
1.0 & $3.28 \times 10^{-7}$ & $2.10 \times 10^{-8}$ & $1.45 \times 10^{-7}$ & $9.32 \times 10^{-9}$ \\
1.5 & $1.24 \times 10^{-6}$ & $7.85 \times 10^{-8}$ & $5.52 \times 10^{-7}$ & $3.49 \times 10^{-8}$ \\
2.0 & $3.43 \times 10^{-6}$ & $2.16 \times 10^{-7}$ & $1.52 \times 10^{-6}$ & $9.61 \times 10^{-8}$ \\
\hline
\end{tabular}

Example (b) Scheme C

\begin{tabular}{lcccc}
\multicolumn{4}{c}{$u_{0}=0, u_{i}=i / p \quad(i=1,2, \ldots, p)$} \\
\hline$x$ & \multicolumn{2}{c}{$p=2$} & \multicolumn{2}{c}{$p=3$} \\
\cline { 2 - 5 } & $h=0.1$ & $h=0.05$ & $h=0.1$ & $h=0.05$ \\
\hline 0.1 & $2.20 \times 10^{-7}$ & $1.38 \times 10^{-8}$ & $8.98 \times 10^{-9}$ & $5.66 \times 10^{-10}$ \\
0.2 & $4.71 \times 10^{-7}$ & $2.95 \times 10^{-8}$ & $3.63 \times 10^{-8}$ & $2.28 \times 10^{-9}$ \\
0.3 & $7.68 \times 10^{-7}$ & $4.81 \times 10^{-8}$ & $8.44 \times 10^{-8}$ & $5.29 \times 10^{-9}$ \\
0.4 & $1.12 \times 10^{-6}$ & $7.04 \times 10^{-8}$ & $1.60 \times 10^{-7}$ & $9.99 \times 10^{-9}$ \\
0.5 & $1.56 \times 10^{-6}$ & $9.75 \times 10^{-8}$ & $2.73 \times 10^{-7}$ & $1.71 \times 10^{-8}$ \\
0.6 & $2.08 \times 10^{-6}$ & $1.31 \times 10^{-7}$ & $4.45 \times 10^{-7}$ & $2.78 \times 10^{-8}$ \\
0.7 & $2.72 \times 10^{-6}$ & $1.71 \times 10^{-7}$ & $7.06 \times 10^{-7}$ & $4.40 \times 10^{-8}$ \\
0.8 & $3.46 \times 10^{-6}$ & $2.17 \times 10^{-7}$ & $1.11 \times 10^{-6}$ & $6.91 \times 10^{-8}$ \\
0.9 & $4.26 \times 10^{-6}$ & $2.68 \times 10^{-7}$ & $1.74 \times 10^{-6}$ & $1.08 \times 10^{-7}$ \\
1.0 & $4.98 \times 10^{-6}$ & $3.14 \times 10^{-7}$ & $2.75 \times 10^{-6}$ & $1.71 \times 10^{-7}$ \\
1.5 & $3.14 \times 10^{-5}$ & $1.90 \times 10^{-6}$ & $3.18 \times 10^{-5}$ & $1.96 \times 10^{-6}$ \\
2.0 & $1.71 \times 10^{-3}$ & $1.05 \times 10^{-4}$ & $5.19 \times 10^{-4}$ & $3.17 \times 10^{-5}$
\end{tabular}




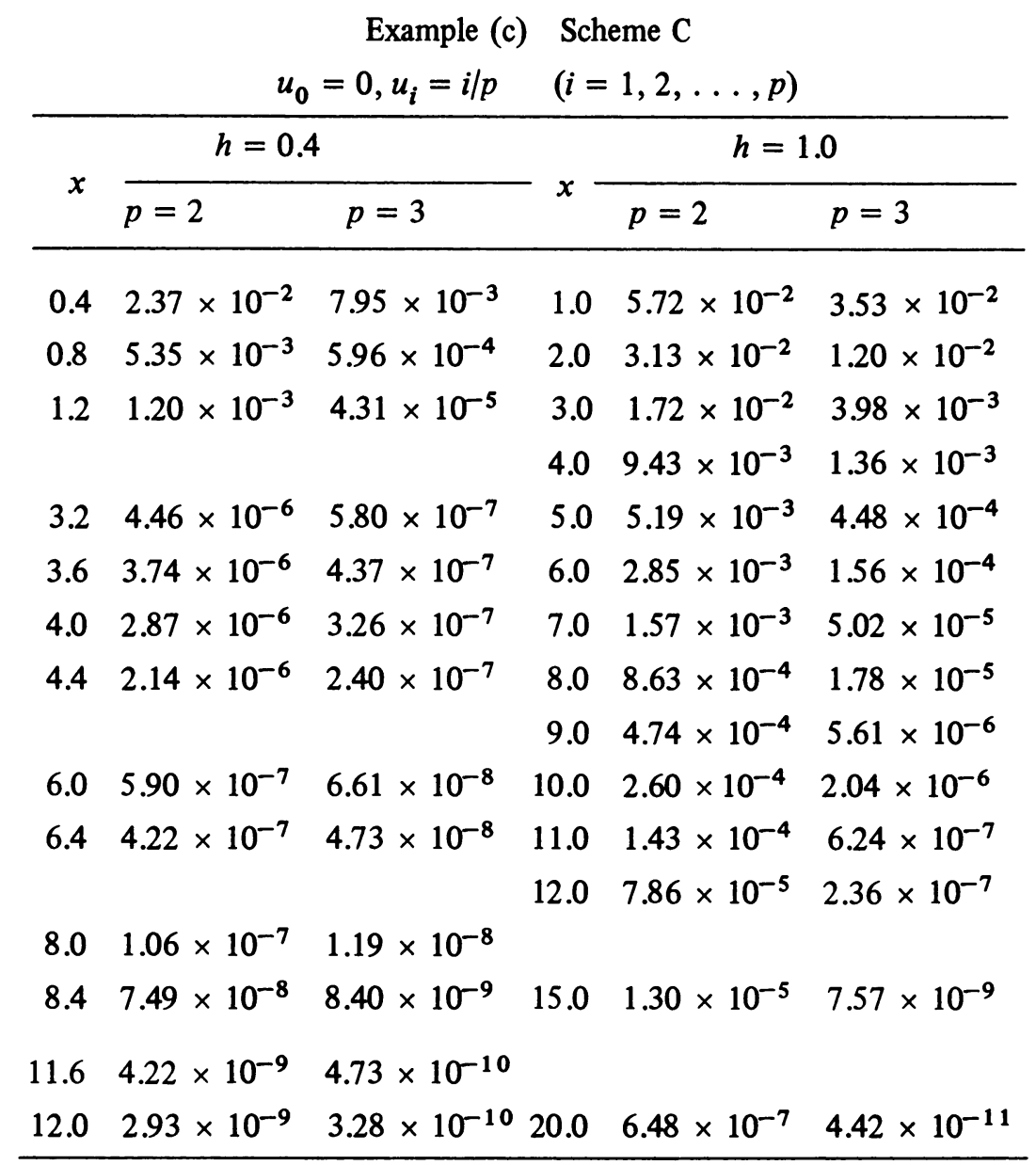

All the above results were obtained on a CDC 7600 and represent absolute errors $y\left(x_{n}\right)-y_{n}, n=0,1, \ldots$

In Makroglou [8], scheme $C$ was tested on one more example for $p=1,2,3,4$, $5, u_{0}=0, u_{i}=i / p, i=1,2, \ldots, p$. In the same reference are also included results with $u_{i}$ being the Lobatto points $\left(p=2, u_{0}=0.5(1-0.2(1+\sqrt{6})), u_{1}=\right.$ $\left.0.5(1+0.2(\sqrt{6}-1)), u_{2}=1\right),\left(p=3, u_{0}=0, u_{1}=0.5(1-\sqrt{0.2}), u_{2}=0.5(1+\sqrt{0.2})\right.$, $\left.u_{3}=1\right),\left(p=4, u_{0}=0, u_{1}=0.5(1-\sqrt{3 / 7}), u_{2}=0.5, u_{3}=0.5(1+\sqrt{3 / 7}), u_{4}=1\right)$. Using these points, order of convergence, higher than the expected from the convergence proof given here, was observed. Weiss [12] has proved an $O\left(h^{p+v+1}\right)$ order of convergence for $e_{m, p}$ in solving equations of the form (1.5) by (1.11) and (1.12). The adaptation of his work to equations of the form (1.1) solved by scheme $C$ will be tried next.

In [8] some examples were also tested for $p=5$ and $u_{i}$ equidistant. This case though, did not seem to have any advantage over the one with $p=4$; for some cases it was even worse in accuracy. This is perhaps due to increased round-off errors because of the increased size of the algebraic equations to be solved. 
Compared with the methods mentioned in the introduction of the same order, tested on the same examples, scheme $\mathrm{C}$ was found the most accurate. Especially on the "stiff" example (example (c)) we obtained very accurate results with errors decreasing as $x$ increases, even with a stepsize as big as $h=1$. For some of the other methods the error grows catastrophically as $x$ increases; see [8, Tables 35,36$]$.

For stability results obtained for the linear test equation $y^{\prime}(x)=\xi y(x)+$ $\eta \int_{0}^{x} y(s) d s, y(0)$ given, $\xi, \eta$ real constants, see [2], [8].

Acknowledgement. This work forms part of the author's $\mathrm{Ph}$. D. thesis written at the University of Manchester under the supervision of Dr. C. T. H. Baker and Dr. Ian Gladwell (for six months), both of whom the author wishes to thank from here too, for their valuable advice, encouragement and criticism.

The author also wishes to acknowledge that the part about convergence with low differentiability requirements (Corollary 1) was added by Dr. Baker.

The M. Sc. and Ph. D. work of the author were supported mainly by the Greek State Scholarships Foundation.

Statistical Unit

Athens University

Panepistemiopolis

Athens-621, Greece

1. O. AXELSON, "A class of $A$-stable methods," $B I T$, v. 9, 1969, pp. 185-199.

2. C. T. H. BAKER, A. MAKROGLOU \& E. SHORT, Stability Regions for Volterra IntegroDifferential Equations, Numerical Analysis Report \#22, Dept. of Mathematics, Univ. of Manchester, U. K., September 1977.

3. H. BRUNNER \& J. D. LAMBERT, "Stability of numerical methods for Volterra integro-differential equations," Computing, v. 12, 1974, pp. 75-89.

4. PH. J. DAVIS \& PH. RABINOWITZ, Numerical Integration, Blaisdell, Waltham, Mass., 1967.

5. J. T. DAY, "Note on the numerical solution of integro-differential equations," Comput. J., v. 9, 1967 , pp. 394-395. York, 1962

6. P. HENRICI, Discrete Variable Methods in Ordinary Differential Equations, Wiley, New

7. P. LINZ, "Linear multistep methods for Volterra integro-differential equations," $J$. Assoc. Comput. Mach., v. 16, 1969, p. 295.

8. A. MAKROGLOU, Numerical Solution of Volterra Integro-Differential Equations, Ph. D. thesis, Univ. of Manchester, U. K., Feb. 1977.

9. W. L. MOCARSKY, "Convergence of step-by-step methods for non-linear integro-differential equations," J. Inst. Math. Appl., v. 8, 1971 , p. 235.

10. K. W. NEVES, "Automatic integration of functional differential equations: an approach," ACM Trans. Math. Software, v. 1, 1975, pp. 357-368.

11. L. TAVERNINI, "One-step methods for Volterra functional differential equations," SIAM J. Numer. Anal., v. 8, 1971, pp. 766-795.

12. R. WEISS, Numerical Procedures for Volterra Integral Equations, Ph. D. thesis, Computer Centre, Australian National University, Canberra, 1972. 